\title{
Web-based Automobile Sales Management System
}

\author{
Huabo Xiao \\ College of Information Engineering, Jiangxi University of Technology, Jiangxi Nanchang
}

Keywords: Sales management system; Logic management; Tomcat; Practicability

\begin{abstract}
The purpose of designing the system is to make the users manage their own sales network only through the internet, change traditional business model and achieve online sales management. The system adopted JSP to write online car sales management system, used Tomcat as the JSP server, connected the system by database SQLServer2000, compiled JavaBean for the logic management of back-office business, which meant to complete the entire design work by JSP + JavaBean + SQLServer2000, and achieved the vehicle management, vehicle classification, car type queries, trading, users order processing, and the administrator system. Practice has proved that this system has strong practicability and value of practical application.
\end{abstract}

\section{Overview}

With the rapid development of economy in our country, the car has gradually appeared in the family, and the ratio of private car ownership is becoming bigger. The rapid development of auto industry has brought new requirements to the sales management, and a new kind of advanced management mode is urgently needed. Increasingly fierce market competition requires enterprises to improve information processing speed, timely and accurately grasp business situation, and make quick business decisions. At present, most of the auto sales management mode is old, and there are many problems in the sales management, which restrict the development and take-off of auto industry. In the traditional auto industry management model, the paper documents and manual management are the mainstream; the problems of this model is poor data reliability, low operation efficiency, difficult for statistical analysis, and remote possibility of extracting useful information for the enterprise. Clearly, how can these shortcomings bring capital and ability to compete with foreign auto industry? The traditional management pattern cannot adapt to the development trend of present auto industry; especially with the arrival of information age, it is practical and important for auto industry members to strengthen their ability, adopt advanced management mode and meet the information revolution.

The system adopted JSP to write web-based car sales management system, connected the system by database SQLServer2000, and compiled JavaBean for the logic management of back-office business, which meant to complete the entire design work by JSP + JavaBean + SQLServer2000. Based on Internet, this system developed the website by JSP language, and paid attention to the interaction ability of users and website. Under such background, web-based auto sales management system has become the preferred architecture in applied software in view of the current development trend of internet. 


\section{System analysis}

Feasibility research. Technical feasibility analysis

The development of online auto management system is complicated system engineering. In order to guarantee the successful development of this system, engineered system development method must be adopted, and some development methods in accord with engineered standards must be studied. These methods are to guide developers for engineered system development so as to accelerate the system development, ensure the quality and reduce development costs. Engineered system development methods have obtained a good result in the practice of development. In the development, JSP was adopted as the development language; Servlet technology was adopted and Tomcat5.0 was the Web server.

Analysis on operation feasibility:

With the popularization and promotion of computer knowledge, more and more people have mastered the basic usage and skills of computer. Along with the development of the Internet, users are familiar with the use of software under the environment of internet and Windows, and show great interest and enthusiasm in new things.

Analysis on economic feasibility:

Online auto system has brought convenience to people, and becomes the brand new business model. Therefore, the new fashion of online home shopping has arrived, and it is completely feasible to construct one online auto management system economically.

According to the above analysis, it's completely feasible to develop online auto management system.

Demand analysis. The task of demand analysis is to make detailed investigation into the objects to be processed in the real world, fully understand the overview of system, clarify various requirements of users, and determine the functions of new system on the basis. The new system must consider the possible extension and changes in future.

Demand analysis on users:

1. User registration

2. User $\log$ in

3. Vehicle model browse

4. Vehicle model query

5. Password settings

6. Personal information settings

7. Comment content

8. Administrator

Analysis on system performance:

The requirement of data security and integrity: confidentiality on user information, which can be seen or searched by administrator, but not be arbitrarily changed. Make sure the security of online payment, and integrity of commodity information and user information; prevent malicious corrections and deletions.

1. High accuracy and reliability

2. Friendly page, complete functions and can be used

3. The system is easy to be maintained and upgraded.

4. High efficiency of access to database

Function analysis. from the aspect of users: 
Through online registration, users can have the following functions: auto model browse, password retrieval, personal information modification, auto overview. After successful registration, users can leave a message on the message board.

From the aspect of website:

1. Website should contain commodity search function: inquire according to the auto fuzzily.

2. Administrator management:

Check user information, and send commodity according to user information and user order.

3. Auto model browse
a).automobile
b).manufacturer
c).model
d). price
e).specification
f).unit
g).quantity

h).explanation

4. Vote for good auto

Check the vote information and score from users.

3.5 Overall structure diagram of system

Separate the manager and client, and make the function more clear

The function of the system is divided into client and manager; the advantages of this division are that the thought is clear and it is convenient for development.

2. three-layer structure

Send requests to business logic layer through uniform interface by adopting three-layer structure user interface layer; business logic layer makes database operation according to its logic rules after processing the requirement, and returns the data returned by database in the form of encapsulating into class to the user interface. User interface only interacts with middle-business logic layer; the database needs no direct operation, and it only needs to maintain the interface with middle-business logic layer. This way improves the database security and maintainability to some extent, and reduces the demand to the developers in the user interface layer.

The division of specific overall system structure diagram is shown in Fig. 1:

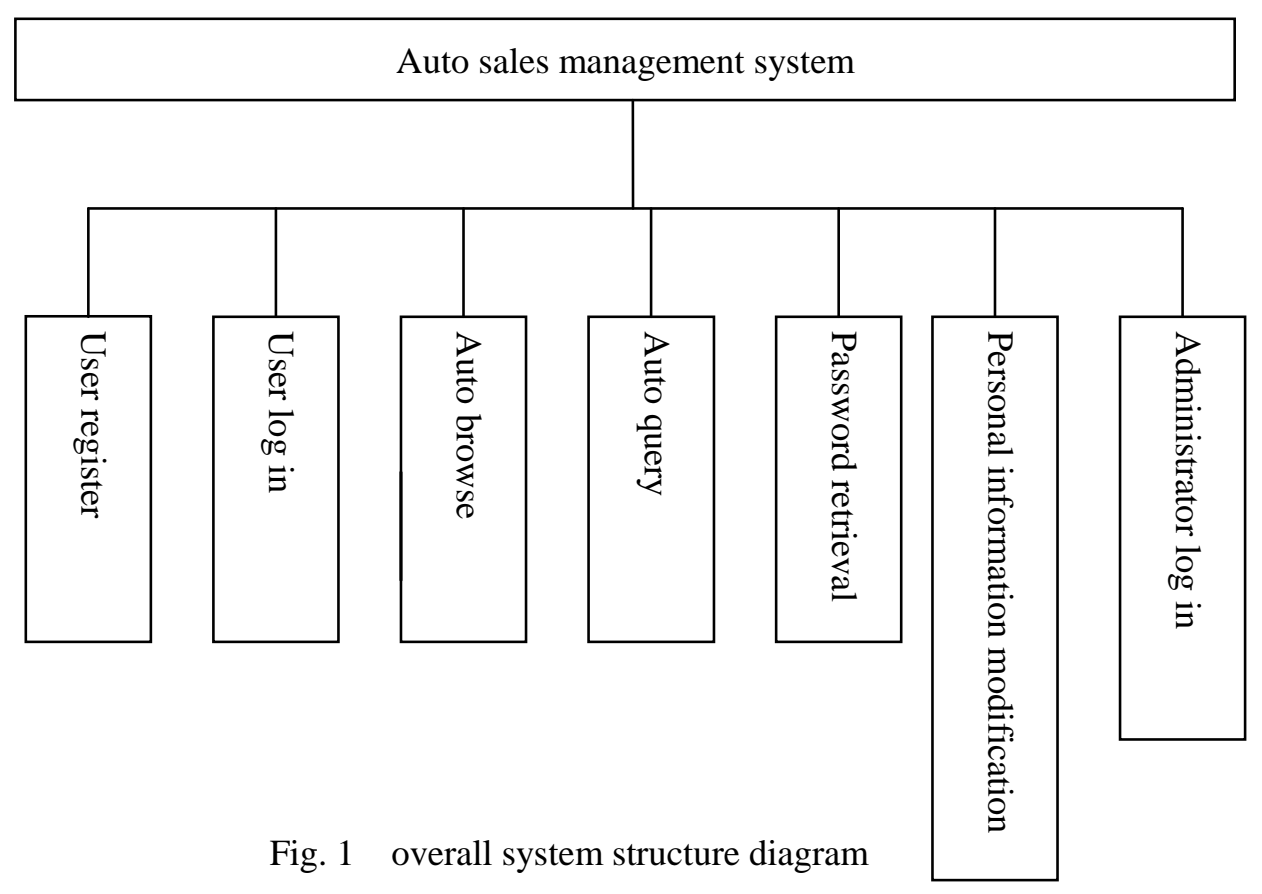




\section{System design}

Whether to establish enterprise internal website to provide service for staffs, commercial website to provide products and service to partners or suppliers, retailing website to sell products or provide service for consumers, or to establish media and entertainment website to release news and other multimedia information, the successful program design should possess good expandability, high-efficiency concurrent processing ability, powerful management tools, integration of existing enterprise information, guarantee of 24-hour service, good fault-tolerance performance, support to a variety of client terminal and safe operation environment.

Design objective. The theoretical design objectives of system function are as follows:

1. Strong practicality: try to make the system be in accordance with the habit of practical operation process, reduce the input of users, make the user interface friendly and easy to learn and use, satisfy the demand of use for each layer;

2. Advanced program structure: the application of contemporary advanced software programming can extend the life cycle, be easy to maintain and management;

3. High security and reliability: complete functions for background maintenance; according to the service condition of the platform in different stages, manager can set corresponding operating authorization, add system registration, distribute the administration privilege of each column, achieve the system maintenance, and guarantee the safety and reliability of the system;

4. The method of using modular design: make the system have good expandability in order to adapt to the development demand of different stages, and be convenient for later analysis and maintenance;

5. Simple operation and convenient maintenance: every sub-system has relatively independent system maintenance function for changeable project;

6. Strong query function: query the basic information on auto and user; query the auto comment information, and make the manager timely and accurately master the basic information of users and commodities.

Module division. According to the demand analysis and design objective of system function and combining with the reality, the function module design of the system can be divided into:

1. Homepage: the main functions and information of the system are shown

2. User registration: register the basic information, in which the phone number and email are important as we process the information for users' payment and mail-order service.

3. User log in: in order to make it convenient for users' payment, mail-order service and management, we can consume only after becoming the member.

4. Auto model browse: after successful log in, users can achieve tabbed browsing of auto model, and submit the satisfied auto into the order page. If the users enter pages directly when they haven't registered or logged in successfully, they will be linked to the "user log in page".

5. Auto model query: users can query the auto they need on the page after successful log-in. If the users enter pages directly when they haven't registered or logged in successfully, they will be linked to the "user log in page". 
6. Password retrieval: users can change their password on the page after successful log-in. If the users enter pages directly when they haven't registered or logged in successfully, they will be linked to the "user log in page".

7. Personal information modification: users can modify the original registration information after successful log-in. If the users enter pages directly when they haven't registered or logged in successfully, they will be linked to the "user log in page".

8. Administrator: check user information and send the commodity according to the users' information.

\section{Database design}

Conceptual structure design of database. Conceptual structure is abstract of real world, namely the artificial process to the real people, object, things and concept; extract the common character concerned by people, neglect the non-essential details and describe accurately these characteristics with various concepts.

According to the above design, the entities include administrator, auto information, and users.

Each entity is the abstract of real objects in reality, and there might be certain relationship among every two or more entities.

The database of the system includes auto model Figure, administrator Figure, guide Figure, comment Figure, individual member Figure. See the details in the following database Figure.

The cross structure of database in JSP. There is standard database access interface similar to the one formulated by Microsoft in JSP; it uses the database access method of Java, which is JDBC, short for Java DataBase Connectivity. JDBC is the platform-independent and standard API of database provided by Sun; it encapsulates the database access into the few methods, and makes the users very convenient to query the database, insert new data and even call stored procedures. In fact, JDBC is a kind of specification; most database vendors provide JDBC driver at present, and the micro-database like Microsoft SQLServer2000 also has several JDBC drivers for choice, which makes the Java application can independently operate in various database. Using the JDBC-ODBC driver developed by Sun, Java can also access the database by ODBC.

The main interfaces provided by JDBC are:

1. java.sq1.DriverManager and Drivermanager are similar to the call-in of processing the driver and provide support to new database link.

2. java.sql.Connection means the connection between applications and specific database.

3. java.sql.Statement is used for common SQL statement execution; SQL statement can query statement, update statement, even create database and execute stored procedures.

4. java.sql.ResultSet queries the returned results and stores in the object, by which the record in the database can be browsed and stored.

If users directly access database in the JSP, java.sql.* has to be introduced before the access. For example, \%@page import="java.sql.*"\%, in this way, JSP can find corresponding SQL interface function.

Establish connection with database. Establish connection with DBMS, the two things must be done: load driver and establish connection.

Load driver: 
The code is needed to load driver. If driven by JDBC driver bridge, the code is as follows:

Class.forName ("jdbc.driver_class_name") ;

When call the Class.forName, it has been automatically loaded. After loading driver, it can be connected with DBMS.

Establish connection:

The second step is to establish connection with DBMS. See the following codes:

Connection con=DriverManager.getConnection(url, "user", "Password")

If use JDBC bridge driver, the URL of JDBC should be jdbc, then the name of data source or database. If the name of database accessed by ODBC is qcgl, the URL of JDBC should be written as jdbc:xjgl. The user name of logging in DBMS should be typed in "myLogin", the latter "myPassword" is the password. The codes are as follows:

Class.forName("com.mysql.jdbc.Driver");

conn=DriverManager.getConnection("jdbc:mysql://localhost/xjgl","root","123");

\section{System implementation}

Module analysis. Make program design according to the previous demand analysis and overall system design content. The system is developed based on Java language. The module is mainly developed and achieved by adopting JSP technology+JavaBeans+ SQLServer2000. All are designed and achieved by the object-oriented means.

In the program design, the following definitions are used and the explanations are as follows:

Page is to define some properties and the value of these properties in the whole JSP page.

The function of Page import is to introduce the class in the JAVA core package and use the class in the program slices part, variable and function declaration part and expression part. The property can be assigned with many values, and the value of the property can be all the classes or one specific class of certain package in JAVA.

Include file means to statically insert one file.

Module division of each function. The home page module

The upside of the home page includes

1. Enterprise member login and registration as well as individual member login and registration

2. Password recovery of enterprise member and individual member

3. Sales information.

4. Buying guide.

5. Website introduction.

6. Auto type and price query.

7. Browse the latest auto type and price.

User registration module

User registration is designed for the users who log in the website for the first time. Users can access other pages of the website only after registration, which includes enterprise member registration and individual member registration.

1. Enterprise member registration includes: login name, password setting, cooperate name, business scope, contact number, etc.

2. Individual member registration includes: login name, password setting, sex, age, etc. 
3. Upon successful registration, users can access other pages of the website.

User login module

1. User login includes: login name, password.

2. Administrator check if the name and password are correct after the users $\log$ in; if they are correct, users can directly access other pages of the website.

Auto information release module

Browse the auto type: upon successful login, users can receive the latest auto model and price through tabbed browsing.

Shopping help page

Don't worry about having no idea on shopping. The Shopping help page will give you a hand.

Functions module diagram.

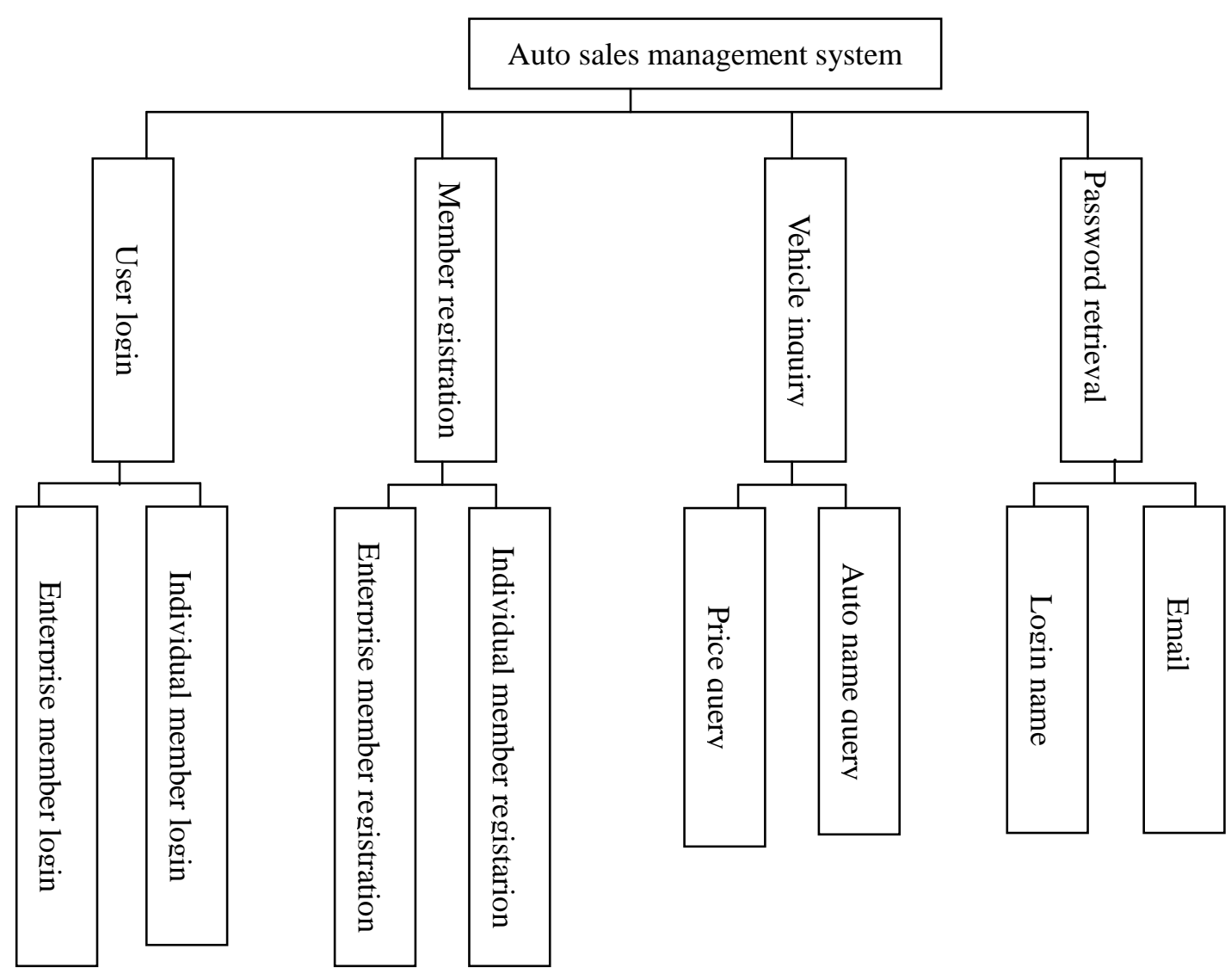

Fig. 2 Figure of system function module of interface

\section{Acknowledgements}

This work was financially supported by project of Jiangxi University of Technology [No.XJXT1403] and Nature of Jiangxi University of Technology [No. ZR14YB03].The project of Technology Department of Jiangxi Province [No 20143BBM26048] also give us a lot of help. 


\section{References}

[1] Wu Yue, Weng Jingnong. Build Web application development strategy with JSP+JavaBean. Volume 42. Year of 2004. The 7th issue. 1181 1193

[2] Zhang Yueping. JSP practical course. Tsinghua University press, March of 2001, 167 182

[3] Liu Yongbo, Liu Xuemei, Zhao Changhai. JSP application development technology. Posts and Telecom Press, Sep, 2005. 21

$\sim 33$

[4] FECIT Technological Product Research Center. JSP application development explained in detail. Electronic Industry Press, May of 2005. 32 36

[5] Liu Yabing, Yang Hong. Be proficient in Eclipse. Electronic Industry Press, June of 2005. $1 \sim 13$

[6] Sun Yong. <Modern software engineering>. Beijing Hope Electronic Press, August of 2003. $12 \sim 15$

[7] Sa Shixuan, Wang Shan. <Introduction to database system>. Higher Education Press, February of 2002. 112 215

[8] Su Zhizhong. Practical guide to DreamweaverMX2004. China Railway Press, May of 2004. $1 \sim 10$

[9] Ji Chengqiang. Dreamweaver is so easy. China Machine Press, April of 2002. 23 30

[10]Yuen, Andy W.K. A Struts Tool for Previewing Forms and Generating Beans. Dr.Dobb's Journal,March, 2004, v 29 ,n 3.59 63 